\title{
Prévention et surveillance de l'addiction à l'alcool et aux stupéfiants : plus de 10 ans d'expérience dans le groupe ExxonMobil
}

\section{Prevention and supervision of addiction to alcohol and drug : more than 10 years experience in the ExxonMobil group}

Patrick CONSTANT*(1), Philippe CORTEEL ${ }^{(2)}$

(1) Directeur Médecine \& Hygiène Industrielle, Centre Expertise Europe/Afrique/Moyen-Orient ExxonMobil Pétrole \& Chimie, Hermeslaan 2 - B-1831 MACHELEN - Belgique (2) Biologiste Toxicologue, 22, rue Champ Lagarde - F-78100 VERSAILLES

* Auteur à qui adresser la correspondance : Patrick CONSTANT, Centre Expertise Europe/Afrique/Moyen-Orient ExxonMobil Petrole \& Chimie, Hermeslaan 2 - B-1831 MACHELEN - Belgique Tél : 32 (0)2 7223800 - e-mail : patrick.constant@exxonmobil.com

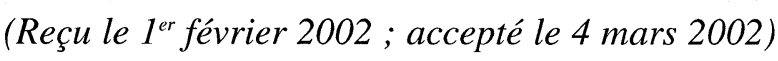

\section{RÉSUMÉ}

Des statistiques inquiétantes et des accidents spectaculaires ont fait réagir le groupe Exxonmobil qui prend maintenant en compte le risque alcool et drogue dans le cadre d'une politique sécurité renforcée. Sensibilisation et information des employés, des partenaires sociaux, définition des postes sensibles ou postes de sécurité, postes où la responsabilité est élevée au plan de la sécurité des personnes, des biens et de l'environnement ont été les premières étapes de la mise en place de cette politique. L'adaptation de cette politique s'est faite dans le cadre de l'aspect réglementaire et juridique français en s'appuyant sur le médecin d'entreprise, seul habilité à prélever, prescrire et recevoir les résultats des tests de dépistage de drogues.

Cette politique fondée sur des protocoles rigoureux et sur une chaîne qualité qui va du recueil des urines jusqu'au

\section{SUMMARY}

Worrying studies and statistics, some catastrophic environmental incidents, conducted ExxonMobil to react, taking into consideration Alcohol and Drug risk within the framework of a reinforced safety policy. Awareness and information of the employees, trade unions, definition of safety positions, designated positions where the responsibility is important versus safety of people, equipment and environment, have been the first steps of policy implementation ; the implementation of an alcohol and drugs $(A \& D)$ policy has been done in a complete compliance with French legal requirements, the occupational physician being one of the key actor only authorized to take samples, to prescribe and to receive the results. This policy based upon specific and strict protocols, on a custody chain, from the biological samples, to the results, needs a complete collaboration between the laboratory and the 
rendu des résultats, nécessite une collaboration étroite entre le médecin du travail, le personnel du laboratoire en charge de recevoir, d'analyser et transmettre les résultats. Audits réguliers et analyses d'échantillons à l'aveugle font partie intégrante de la chaîne de qualité, ce qui assure une garantie absolue au médecin, à la personne testée, à l'entreprise. Le laboratoire doit avoir des procédures écrites correspondant aux exigences du GBEA et assurer un contrôle de qualité interne et externe. Les étapes analytiques principales sont les suivantes : recherche d'adultérants, screening par immunoanalyse, confirmation par chromatographie en phase gazeuse/spectrométrie de masse, validation et contrôle des résultats.

Une telle politique doit s'inscrire dans le cadre d'un programme de sécurité, et sa mise en place exige une adhésion de tous les acteurs : employés, direction, partenaires sociaux. Le médecin du travail doit jouer un rôle majeur, essentiel comme expert et garant de l'éthique médicale et de la confidentialité.

Même si, à la lumière des résultats collectés pendant plus de 10 ans, l'usage de drogues en milieu de travail est relativement limité, il est indispensable de prendre en compte le risque alcool et drogue dans le cadre d'une politique globale sécurité.

\section{MOTS-CLÉS}

Drogue, médecin d'entreprise, laboratoire.

\section{Introduction}

Des statistiques et études inquiétantes ont fait réfléchir le groupe EXXONMOBIL, des accidents spectaculaires et catastrophiques sur le plan environnement l'ont fait réagir, prenant en compte le risque alcool et drogues (A\&D) dans le cadre d'une politique sécurité renforcée. Aux États-Unis, $20 \%$ de la population génèrale et $10 \%$ de la population active usent de drogues, $25 \%$ des chauffeurs tués dans les accidents de la circulation étaient positifs à la cocaïne (1), $30 \%$ des conducteurs impliqués dans un accident de la circulation sont positifs au THC $(2,3)$ et $39 \%$ des conducteurs arrêtés pour infraction sont positifs au THC (4).

En France, on recense environ 400000 toxicomanes (60\% au Cannabis et $30 \%$ à l'héroïne). Une étude nationale conduite en 1989/1990 révèle $8 \%$ de résultats positifs aux benzodiazépines et au Cannabis chez des conducteurs victimes d'accidents graves ou mortels, alors que $14 \%$ des chauffeurs blessés dans des accidents de la circulation sont positifs au THC (5). Plus récemment en France, on avancerait le chiffre de $30 \%$ de conducteurs impliqués dans des accidents mortels sous influence du Cannabis et/ou de l'alcool.

L'abus d'alcool et d'usage de drogues ont un effet négatif sur la santé et la sécurité, mais également sur l'efficacité et la productivité dans le monde économique et industriel. Tous ces éléments ont conduit il y a plus de occupational physician.

Regular audits, blind samples program, included in the custody chain, reinforce the safeguard of the lab staff, physician, employee, and employer. The lab must have written protocols aligned with the GBEA requirements ensuring an internal and external Quality Control. The main analytical steps are the following: check for diluted or adulterated specimens, screening by immunoassay, confirmation by GC/MS, control of the results.

An A\&D policy must be a part of a Safety program, the implementation needs an agreement of all the actors, employees, trade unions and management. The occupational physician is a key actor as an expert and guarantor for medical confidentiality and ethics.

Even if drug abuse is limited, at the light of ten years of experience, the drug and alcohol abuse issue must be managed to assure to all employees a safe, healthy and productive workplace within the framework of a global safety policy.

\section{KEY-WORDS}

Drug policy, occupational physician, laboratory.

dix ans, la société Exxonmobil à mettre en place une politique A\&D. Dans le cadre de cette politique A\&D, le dépistage des conduites toxicophiles, aussi bien à l'embauche qu'au cours de l'activité professionnelle, prend une place importante. L'adaptation du modèle américain à la France nécessite le rôle actif du médecin du travail.

\section{Les étapes de la mise en place d'une politique "drogue"}

Les prémices de la mise en place d'une politique «drogue» passent par un effort de communication pour sensibiliser et informer le personnel et les partenaires sociaux. Cette surveillance ne s'applique qu'aux personnels qui occupent des postes sensibles ou postes de sécurité définis comme suit : a) poste où la responsabilité est élevée au plan de la sécurité des personnes, des biens et de l'environnement ; b) poste où le titulaire n'a pas ou peu de supervision et joue un rôle direct dans une tâche opérationnelle.

Les postes sécurité, au sein du groupe Exxonmobil, représentent $10 \%$ de la population. Ces postes sont surveillés systématiquement au regard de la consommation d'alcool et drogues. 


\section{Les facteurs de succès d'une politique "alcool \& drogues"}

La politique A\&D doit être comprise et acceptée par l'ensemble des employés et des partenaires sociaux. Elle doit s'inscrire dans le cadre d'un programme plus large de sécurité, en restant en conformité avec le cadre réglementaire et juridique français. Elle doit prendre en compte les aspects de prévention, d'information, de surveillance et de suivi, dans le cadre d'un programme "cure" et "post cure". Enfin, le médecin d'entreprise doit être le pivot de cette politique, et il en est l'acteur clef du succès de sa mise en place.

\section{Rôle du médecin du travail}

Il définit le panel de drogues à dépister et les seuils de positivité. Le panel exploré chez Exxonmobil est le suivant : amphétamines, barbituriques, benzodiazepines, cannabis, cocaïne, méthadone, opiacés, phencyclidine et propoxyphène.

Il conduit les examens médicaux et établit les procédures de recueil des échantillons avec le laboratoire choisi.

Il sélectionne le laboratoire, s'assure de la mise en place d'une chaîne de qualité, allant du recueil des échantillons jusqu'au rendu des résultats. Il conduit des audits réguliers du laboratoire, pratique des tests de qualité en envoyant des échantillons tests.

Il prescrit, reçoit les résultats, les analyse, et au terme d'une enquête minutieuse (prise de médicaments pouvant expliquer un résultat positif), finalise sa décision après s'être assuré du respect de la chaîne de qualité. Enfin, il met en place un programme de cure de désintoxication et de post-cure. Il participe aux actions régulières de sensibilisation et de prévention et il est le garant de l'éthique médicale et de la confidentialité.

\section{Mise en pratique}

Les tests sont pratiqués à l'embauche systématiquement pour tout poste de sécurité, avant la mutation vers un poste de sécurité, et de façon régulière (au moins une fois par an) pour les titulaires de poste de sécurité, et enfin dans le cadre d'un suivi post-cure. Ces tests, à l'exception du suivi post-cure, sont intégrés dans le cadre de la visite médicale.

Le recueil des urines après information du patient (lettre de consentement), répond aux exigences de la chaîne qualité. Les urines sont recueillies dans deux récipients, scellés, codés et ensuite contrôlés par l'employé. Un échantillon est adressé de façon anonyme au laboratoire ; le deuxième flacon est congelé et mis de côté si un contrôle de résultats est demandé par l'intéressé ou le médecin. Le médecin est le seul à recevoir les résultats du laboratoire.

\section{Rôle du laboratoire}

Le biologiste doit avoir rédigé un protocole éprouvé et testé, dont il doit être capable de vérifier toutes les étapes. Ce protocole décrit toutes les étapes pré-analytiques, analytiques et post-analytiques conformément au GBEA (guide de bonne exécution des analyses); ce protocole est à la disposition du médecin du travail qui peut en demander modification après consultation auprès d'un expert autorisé à agréer ou non le laboratoire et le biologiste.

Les étapes pré-analytiques doivent permettre de s'assurer :

- De l'intégrité de l'emballage

- Du délai d'acheminement

- De la conformité de l'étiquetage du flacon avec les données du formulaire de la chaîne de qualité rempli par le médecin.

- De l'intégrité du système de fermeture du flacon.

- D'un enregistrement exact au niveau du secrétariat

- Du stockage de l'échantillon dans de bonnes conditions et dans des locaux appropriés.

L'étape analytique comporte :

- L'étude des caractères suivants : couleur, odeur, pH (refuser l'échantillon si $\mathrm{pH}>8,5$ ), densité et créatinine (pour s'assurer qu'il s'agit bien d'une urine : on ne retient que les échantillons dont la concentration en créatinine est supérieure à $0,50 \mathrm{~g} / \mathrm{l}$ ).

- La recherche des facteurs adultérants : dilution, addition d'eau de Javel, addition de sel, addition de glutaraldéhyde.

Si toutes les étapes précédentes sont satisfaisantes, on passe au screening c'est-à-dire à la recherche par immunoanalyse des principes actifs ou des métabolites. Le choix du réactif est important et dans certains cas on doit utiliser deux réactifs différents (anticorps monoclonaux et polyclonaux), pour une même famille de produits. L'appareil est régulièrement vérifié. Dans chaque série doivent-être inclus des témoins négatifs et positifs.

Un résultat supérieur au seuil est déclaré positif, mais si une réaction donne une D.O. inférieure de $10 \%$ à celle du seuil, le résultat est qualifié de douteux. Pour les urines négatives, l'étape analytique est terminée

L'étape de confirmation est obligatoire pour affirmer la 
positivité. Elle utilise la chromatographie en phase gazeuse couplée à la spectrométrie de masse (CG/SM). Elle nécessite une extraction rigoureuse, une dérivation et une étude du spectre de masse. Tout comme pour le screening, on inclut dans la série une urine positive et une urine négative. Si le résultat met en évidence le principe actif ou un métabolite, on confirme le résultat du screening.

Dans certain cas, on ne retrouve ni principe actif ni métabolite : on conclut à une réaction de dépistage faussement positive. On doit cependant identifier le produit qui a positivé la réaction de screening (par exemple la chloroquine croise avec les amphétamines.)

L'étape post-analytique comporte :

- La validation des résultats par un biologiste compétent en toxicologie qui s'assure de la correcte traçabilité de toutes les étapes.

- La rédaction du compte rendu : celui ci ne comporte pas de nom, mais le numéro indiqué par le médecin, afin de préserver la confidentialité. Le compte rendu doit clairement définir le produit en cause et sa concentration. Il doit stipuler le respect de toute la chaîne de qualité.

Il doit-être accompagné d'un commentaire du biologiste qui signe les résultats. Si la fausse positivité est due à la prise d'un médicament, il doit s'assurer que l'employé l'avait bien consommé.

- L'expédition par poste sous pli confidentiel au médecin prescripteur.

- Les urines positives sont conservées à $-20^{\circ} \mathrm{C}$ pendant un an pour répondre à une demande de contrôle éventuel aussi bien de la part du médecin prescripteur que de l'employé.

- Le biologiste doit-être capable de fournir au médecin la preuve de traçabilité des différentes étapes.

\section{Contrôles de qualité}

Le biologiste toxicologue doit pratiquer de façon régulière des contrôles de qualité internes et externes. Le médecin du travail peut envoyer des urines tests dont il connait la composition pour vérifier la qualité du laboratoire. Par cette procédure le médecin du travail assure une qualité pour lui-même, l'employé et l'employeur.

\section{Conclusion}

Une politique A\&D doit s'inscrire dans le cadre d'un programme sécurité, et sa mise en place exige une adhésion de tous les acteurs : employés, directions, partenaires sociaux. Le médecin du travail doit jouer un rôle majeur, essentiel comme expert et garant de l'éthique médicale et de la confidentialité. En liaison avec les responsables du laboratoire de toxicologie pratiquant les analyses, il doit s'assurer que la chaîne qualité est respectée, du recueil des urines jusqu'au rendu des résultats. Même si, à la lumière des résultats collectés pendant plus de 10 ans dans une grande entreprise comme Exxonmobil, l'usage de drogues en milieu de travail est relativement limité, il est indispensable de prendre en compte ce risque dans le cadre d'une politique globale sécurité.

\section{Références}

1. Marzuk P.M., Tardiff K., Leon A.C.,Stajic M.,Morgan E.B.,Mann J.J. Prevalence of recent cocaine use among motor vehicle fatalities in New York City. JAMA,1990 ; $263: 250-6$.

2. Skolnick A. Illicit drugs take still another toll : death or injury from vehicle associated trauma. JAMA, 1990 ; $263: 22-5$.

3. Soderstrom C.A. Drug involvement among drivers admitted to regional center. Transportation research board, 70th annual meeting, Washigton DC January 15th , 1991.

4. Sutton L.R., Paegle I. The drugs impaired driver detection of forensic specimen analysis. Blutalkohol, 29 (2) : 134-8-92.

5. Blanquard D., Coudane H., Aussedat M., Peton P., Pagel E., Niziolek S. Influence de la consommation de Cannabis sur les accidents de la voie publique. J.Méd. Lég. Droit Med., 1989 ; 287-90. 